\title{
In-Situ Probing of the Biotic-Abiotic Boundary of Plants by Laser Desorption/Ionization Time-of-Flight Mass Spectrometry
}

\author{
Chanan Sluszny, and Edward S. Yeung \\ Ames Laboratory-United States Department of Energy and Department of Chemistry, Iowa State University, \\ Ames, Iowa, USA \\ Basil J. Nikolau \\ Department of Biochemistry, Biophysics, and Molecular Biology, Iowa State University, Ames, Iowa, USA
}

\begin{abstract}
Laser desorption/ionization time-of-flight (LDI-TOF) mass spectrometry was applied for the direct analysis of cuticular waxes on intact plant tissues. Cuticular wax compounds were ionized by laser desorption in the presence of colloidal silver. Silver-adduct ions were detected on samples from Arabidopsis thaliana and from maize. Good spot-to-spot reproducibility indicated homogeneous coverage of the sample by the fine colloidal material. The results were consistent with GC-MS analyses of cuticular extracts, thus confirming the feasibility of direct analysis based on this protocol. Molecular masses of the adduct ions correspond well with the known composition of cuticular waxes. Moreover, LDI-TOF gave good estimates of the relative local abundances of a given compound. However, bias was found in cases where compounds with different ionization efficiencies were analyzed. (J Am Soc Mass Spectrom 2005, 16, 107-115) (c) 2004 American Society for Mass Spectrometry
\end{abstract}

$\mathrm{T}$ The outer tissue matrix of an organism or cell is critical to the boundary between the biotic world and the abiotic environment. In plants this boundary is the cuticle, and its biochemical composition, structure, and biogenesis are still poorly understood. The cuticle, which coats the aerial surface of plants, functions as a water-barrier. It is composed of cutin, a lipid-based polyester, which is intermixed with cuticular waxes [1]. These are known to be composed of various long-chained alkanes, aldehydes, ketones, fatty acids, alcohols, and esters [2]. Although the identity of the gene products involved in the production of cuticular waxes is starting to emerge, other studies in this field remain enigmatic [2]. Research in this area is usually based on visual screening, which is employed to identify genetic lines with an altered cuticle [2, 3]. The cuticular waxes of the selected samples are usually extracted and analyzed by gas chromatography mass spectrometry (GC-MS). Such screening procedures are usually laborious and inefficient.

Laser desorption/ionization time-of-flight (LDI-TOF) mass spectrometry has been used for the analysis of various long-chain nonpolar hydrocarbon compounds [4-7]. Analysis is usually based on the co-deposition of transition metal salts or powders on the sample [4-7]. The

Published online December 15, 2004

Address reprint requests to Professor E. S. Yeung, Ames LaboratoryUSDOE and Department of Chemistry, Iowa State University, Ames, IA 50011, USA. E-mail: yeung@ameslab.gov likely mechanism is that absorption of laser light by the additive leads to vaporization of the mixture and the formation of gaseous metal ions. Attachment of metal ions to the analyte in turn produces ionized species that can be analyzed with a mass spectrometer. The mechanism is different from that in matrix-assisted laser desorption ionization (MALDI) [8, 9], where the matrix absorbs as well as transfers protons to the analyte to produce ions. The phenomenon is also different from that based on ultra-fine metal powder $[10,11]$ (UFMP). By itself, UFMP produces ions from intact polymers with moderate molecular weight. When mixed with glycerin, even high molecular weight polymers can be ionized without fragmentation. However, in neither case was the metal-ion adduct observed. Transition metal-ions have been successfully used in mass-spectrometric characterization of polymers [12], technical and microcrystalline waxes [4, 6], and various lipid compounds [5, 7]. In this approach, it has been shown that silver is the most promising among ionization agents for these compounds [5]. Chen and Li [5] found that the LDI-TOF spectrum of long-chain alkanes obtained by using silver powder displayed low fragmentation. Dutta and Harayama [7] got similar results by using silver nitrate for the chemical ionization of various alkanes and crude oil samples.

The main disadvantage of the co-deposition methodology is the inhomogeneous distribution of the additive on the sample. Consequently, one needs to search for the "sweet spots" on the sample target in order to obtain good mass spectra. Attempts have been made to 
improve the reproducibility of ionization. In particular, solvent-free sample preparation $[6,13,14]$ has been applied successfully for the characterization of paraffins and waxes [6], and for the analysis of polymers with the addition of typical matrixes used in MALDI [13, 14]. This operation, which is based on mixing powders of analyte, metal additive, and matrix, requires large amounts of sample and can only be used when spatial information is not sought.

One important application of LDI-TOF is the direct analysis of compounds from a cell or tissue sample without extraction or separation [15]. For example, MALDI-TOF has been recently used for the direct profiling of peptides and proteins in single cells or tissues [15-18]. However, since LDI-TOF is primarily a surfaceprobing tool, e.g., ions are generated only from the exterior regions of the sample that are exposed to laser irradiation [19], determination of intracellular contents usually requires special sample preparation [15]. In this study, LDI-TOF is used for the direct analysis of the plant surface. Analysis becomes straightforward since cuticular waxes are found on the outer surface layer of plants (0.1-1 micron) [20]. LDI-TOF offers rapid screening of multiple samples and could therefore become a valuable tool in analysis of the cuticle and various other compounds related to plant metabolomics.

\section{Experimental}

\section{Chemicals}

Various n-alkanes, alcohols, esters, ketones, and fatty acids, consisting of 13-32 carbon atoms, were purchased form Sigma-Aldrich (St. Louis, MO). A mixture of n-alkanes $\left(\mathrm{C}_{12}-\mathrm{C}_{60}\right)$ was obtained from Supelco (Bellefonte, PA). This mixture contained $85 \mu \mathrm{g} / \mathrm{mL}$ of each compound in cyclohexane. Silver nitrate and silver powder were purchased from Aldrich (St. Louis, MO). Colloidal suspension of silver (20 ppm) was purchased from Purest Colloids (Westhampton, NJ). Tetrahydrofuran (THF) was obtained from Fisher (Pittsburgh, PA). Double-deionized water was used in all cases for sample preparation.

\section{Sample Preparation}

Lipids were dissolved in THF to a final concentration of $0.75 \mathrm{mg} / \mathrm{mL}$. Silver nitrate was dissolved in water to a final concentration of $5-10 \mathrm{mg} / \mathrm{mL}$. Silver powder was prepared in 2-propanol. One to $2 \mu \mathrm{l}$ of lipid sample was deposited on a commercial MALDI target-plate and air-dried. Then, $2 \mu \mathrm{l}$ of the metal additive (salt, metal powder, or colloidal suspension) was deposited onto the sample and dried under vacuum. For colloidal silver, an additional $2 \mu \mathrm{l}$ were added in some cases in order to increase its final amount on the sample. Cuticular waxes from Arabidopsis thaliana, ecotype Columbia, were extracted with chloroform.

Plants of Arabidopsis thaliana, ecotype Columbia, were grown in a controlled environment at $21-23{ }^{\circ} \mathrm{C}$ with a $24 \mathrm{~h}$ photoperiod. Plants of various ages were analyzed. Maize inbred B73 seedlings were grown in a greenhouse at $31{ }^{\circ} \mathrm{C}$ with a $24 \mathrm{~h}$ photoperiod. Thin transverse slices of Arabidopsis thaliana stems were cut with a razor blade. Samples from Arabidopsis thaliana leaf and from maize seedling leaves were cut with a razor blade. All samples were dried in moderate vacuum (approximately 50 torr) for 30-60 min. Plant material was then cut into $2 \mathrm{~mm}$ segments and attached onto the surface of a target plate with double-sided tape. Metal additive was then applied onto each sample with a pipet. The surface of the double-sided tape helped in keeping the added solution (or colloids) on top of the plant sample. In most cases $5 \mu \mathrm{L}$ was sufficient for complete coverage of plant material by the metal additive. Subsequently, samples were dried in vacuum. For silver colloids, multiple additions (5-6 additions of $5 \mu \mathrm{L}$ ) to the plant material were employed in order to achieve good surface coverage. A target plate with the attached tissue sample, prepared for the mass-spectrometric analysis, is shown in Figure 1.

\section{TOF Mass Spectrometry}

Mass-spectrometric analysis was performed with a Biosystems Voyager-DE PRO instrument (Framingham,

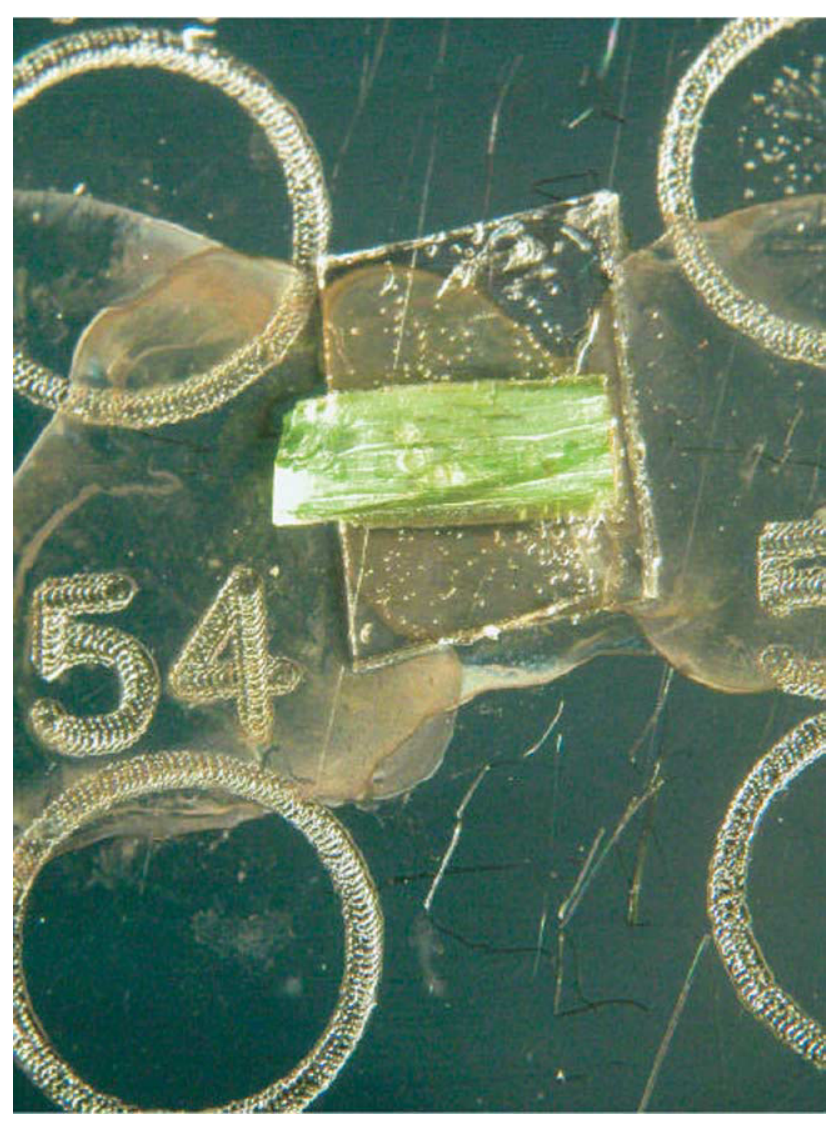

Figure 1. Stem tissue of Arabidopsis thaliana on a MALDI target plate. Sample was covered with colloidal silver for mass-spectrometric analysis. 
MA) equipped with a $\mathrm{N}_{2}$ laser (337 nm, 3-ns pulse width, $3-\mathrm{Hz}$ repetition rate, $180 \mu \mathrm{J}$ maximum energy per pulse). The mass spectra were acquired in the reflectron mode with a delayed extraction period of 100 ns. Positive ions were subjected to a $20 \mathrm{kV}$ accelerating potential. Typically, 100-200 laser pulses were acquired for each measurement. External mass calibration was performed with a $\mathrm{C}_{12}-\mathrm{C}_{60}$ alkane standard mixture.

\section{Scanning Electron Microscopy}

$1 \mu \mathrm{L}$ of colloidal silver was deposited onto a flat silicon surface. After drying, the spotted area was approximately $2 \mathrm{~mm}$ in diameter. The sample was then attached onto a double-sided carbon dot and coated with carbon (Cressington 308R-EM vacuum evaporator, Cressington, Cranberry, PA). Samples were analyzed with a scanning electron microscope (AmRay model 1845FE, Amray, Bedford, MA).

\section{Results and Discussion}

\section{Colloidal Silver Additive}

Determination of long-chain non-polar hydrocarbons by LDI-TOF is based on either mixing or co-deposition of a metal ionization agent on the sample [4-7]. Irregular deposition of metal ions or particles on the sample is a known problem of this methodology [6], leading to "sweet spots" on the target. Improvement in spot-tospot reproducibility is therefore dependent on uniform and complete coverage of the sample target with the ionization material. This is difficult to achieve with silver nitrate or silver powder [4-7]. Silver nitrate usually forms large crystals upon solvent evaporation. Ionization is therefore limited to the regions proximal to the deposited crystals. To date, application of silver powder has not provided much better performance, mainly due to the large dimensions of the particles $(\sim 1$ $\mu \mathrm{m})$.

Colloidal materials, on the other hand, are highly stable in aqueous suspension and do not produce bulk crystals upon solvent evaporation. Colloids should therefore provide relatively homogeneous surfacecoverage. This is illustrated in Figure 2, which is a scanning electron microscopy (SEM) image of silver colloids on a flat surface. As may be expected, some void spaces are found at low concentrations of the additive (Figure 2a). These void regions can be eliminated by multiple additions of the colloidal material or by using a higher concentration of colloids (Figure 2b). At the higher magnification (Figure 2c), formation of aggregates becomes evident. These colloidal aggregates, however, appear to be randomly distributed over the surface.

For the determination of standard lipid mixtures, a single addition of silver colloids was sufficient in most cases and good spot-to-spot reproducibility was achieved. In the multiple addition procedure, a large amount of silver colloids can be accumulated on the sample from a low-concentration solution, thus improving surface coverage. In each step, colloids were added onto the sample, which was then dried in vacuum. Spot-to-spot reproducibility was significantly improved by using the multiple addition procedure, in particular for the direct analysis of plant tissue. Differences in signal intensities measured at the various locations on
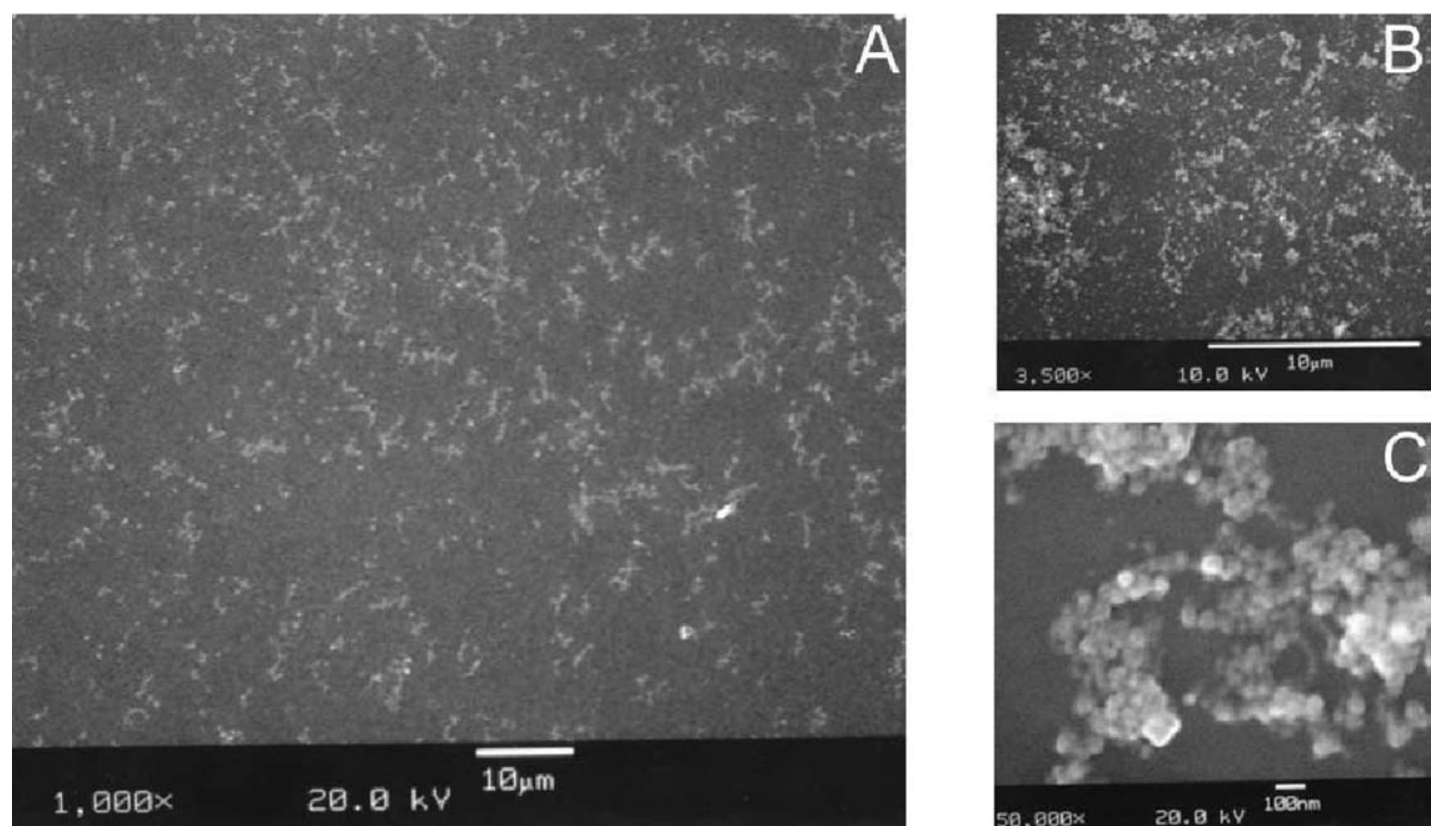

Figure 2. Scanning electron microscopy image of colloidal silver on a flat surface produced by using (a), a moderate concentration of colloids; (b), a concentrated suspension of colloids; and (c), same as (a) except at a higher magnification. 


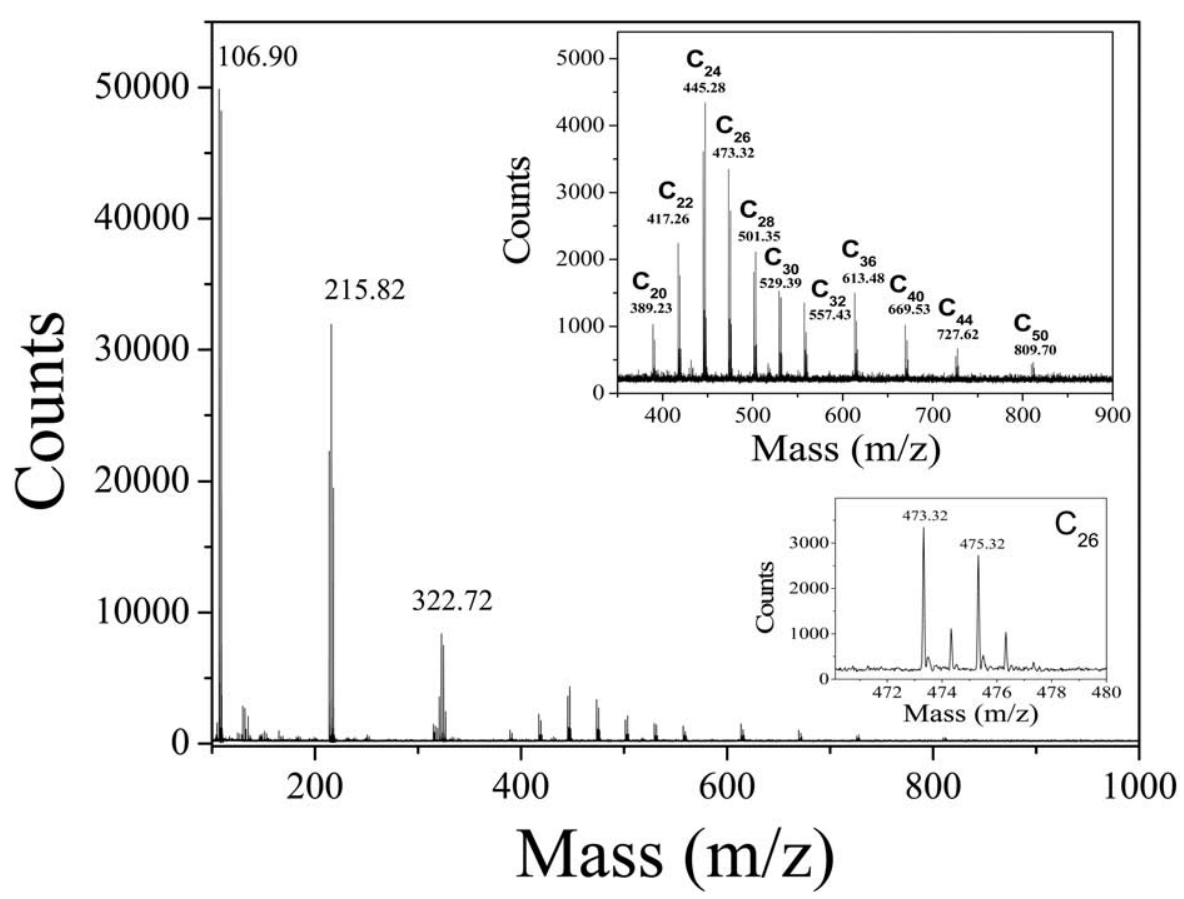

Figure 3. LDI-TOF mass spectrum of the $\mathrm{C}_{12}-\mathrm{C}_{60} \mathrm{n}$-alkane standard mixture as silver adducts. The top inset is the enlarged view of the region $350-900 \mathrm{~m} / \mathrm{z}$. The lower inset is the enlarged view for the $\mathrm{C}_{26}$ alkane.

the target or tissue, vide infra, should thus reflect the local concentrations of the analytes.

\section{Mass Spectra of Standard Hydrocarbon Mixtures}

n-Alkane compounds can account for approximately $40 \%$ of total content in cuticular wax [2, 3, 21,22]. These compounds, which are commercially available as highpurity mixtures, were therefore chosen for the initial experiments with colloidal silver additive. This same mixture was later used for external mass calibration in the analysis of wax compounds.

A typical mass spectrum of the silver-adduct ions of a standard $\mathrm{C}_{12}-\mathrm{C}_{60} \mathrm{n}$-alkane mixture is presented in Figure 3 . The mass accuracy for the various compounds in the mixture was approximately $20 \mathrm{ppm}$. Silver ions and clusters dominate the low-mass region of Figure 3. Peaks of n-alkane compounds can be discerned with masses higher than approximately $400 \mathrm{~m} / \mathrm{z}$. Thus, the analysis of $n$-alkanes is not affected by the ionization additive. The lower inset of Figure 3 shows an enlarged view for the region around $C_{26}$. The two major peaks correspond to the ${ }^{107} \mathrm{Ag}$ and ${ }^{109} \mathrm{Ag}$ adduct ions and the two minor ones are related to carbon and hydrogen isotopes. This LDI-TOF mass spectrum is typical of n-alkane compounds [7].

The results in Figure 3 indicate large differences in the ionization efficiencies of the various n-alkanes. In particular, the high intensities of the $\mathrm{C}_{22}-\mathrm{C}_{28}$ nalkanes are in stark contrast to the lack of any signal from alkanes smaller than $\mathrm{C}_{20}$. Dutta and Harayama
[7] obtained similar results by using silver nitrate as an additive. They found that $\mathrm{C}_{28}$ exhibited the highest ionization efficiency and compounds smaller than $\mathrm{C}_{24}$ could not be detected. This difference in ionization efficiencies for the various n-alkanes is not well understood [5]. We found, however, that certain experimental conditions, such as laser power, can affect the ion signals. For example, $C_{20}$ and $C_{22}$ became undetectable at high laser power while $\mathrm{C}_{60}$ produced a measurable signal. Nevertheless, as long as the experimental procedures are not altered, the ionization efficiency for a particular n-alkane remains relatively constant.

Quantitative estimate of the various lipids in a mixture is more difficult. This is because of the dependence of ionization efficiencies on the specific compounds, as is shown in Figure 4. There, the concentrations (wt/vol) of all compounds in the sample were identical. However, each compound produced a different signal intensity. In particular, we note the high ion signal from $C_{32}$ ester and the low ion signal from n-alkane. Additional measurements (data not shown) of various long-chain lipids supported the findings of Figure 4. Quantitative estimates of the components in cuticular wax mixtures will depend on having standards for each class of compounds. However, pure standard mixtures of a broad spectrum of lipids are not commercially available. Therefore, the experiments here can only provide estimates of the relative abundances of individual compounds in different samples. 


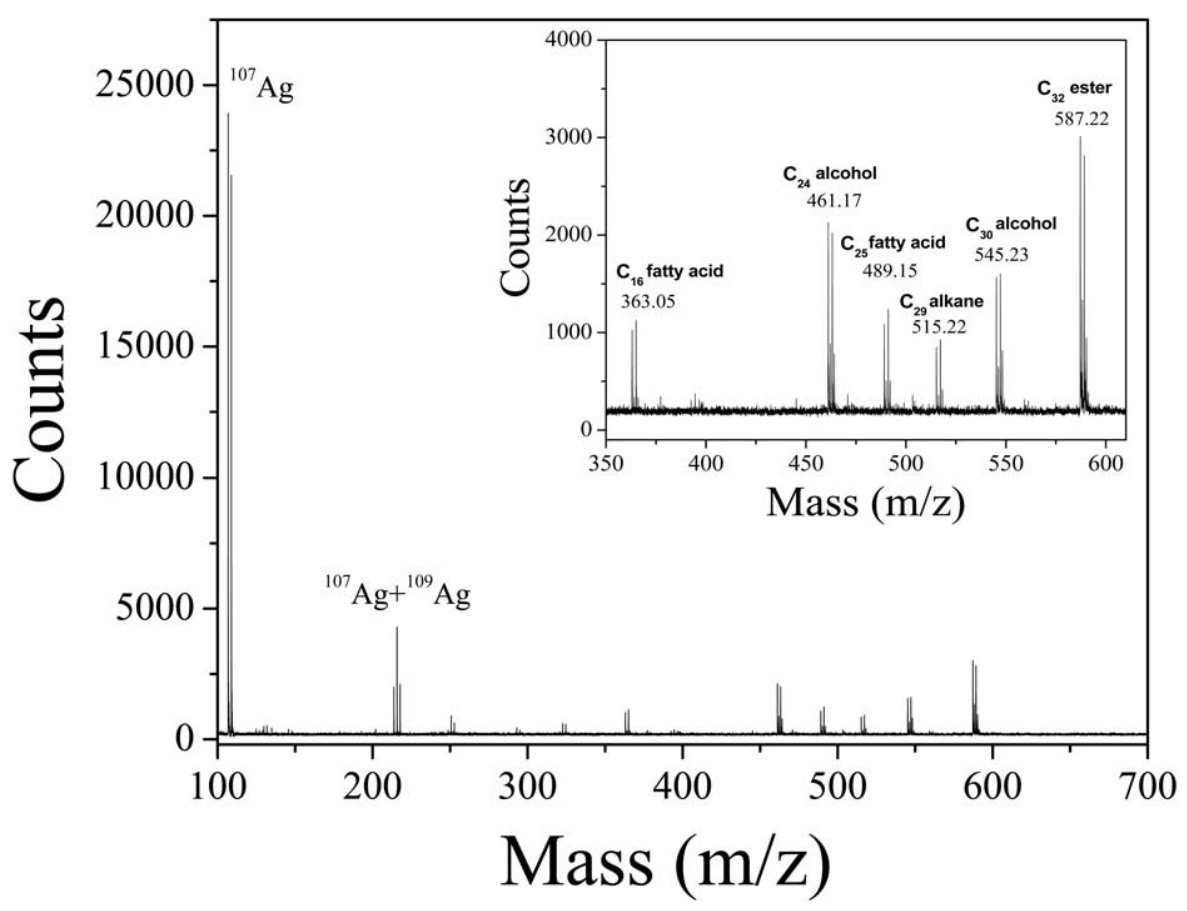

Figure 4. LDI-TOF mass spectrum of a mixture of long-chain hydrocarbon compounds as silver adducts. The inset is the enlarged view of the region $350-600 \mathrm{~m} / \mathrm{z}$.

\section{Analysis of Cuticular Waxes on Plant Tissue}

In this study Arabidopsis thaliana was selected as the model plant target for direct analysis of cuticular waxes. Composition of wax in Arabidopsis thaliana is well documented so that an evaluation of the performance of LDI-TOF is possible. In this section, results of the analysis of stem tissue are presented first. Results of direct LDI-TOF analysis of Arabidopsis thaliana leaves and for maize are presented at the end of this section.

Samples of Arabidopsis thaliana cuticular wax extracts and of whole plant tissue were analyzed separately by using LDI-TOF with silver colloid as an additive. The results for the whole plant tissue are presented in Figure 5. The mass accuracy was ap-

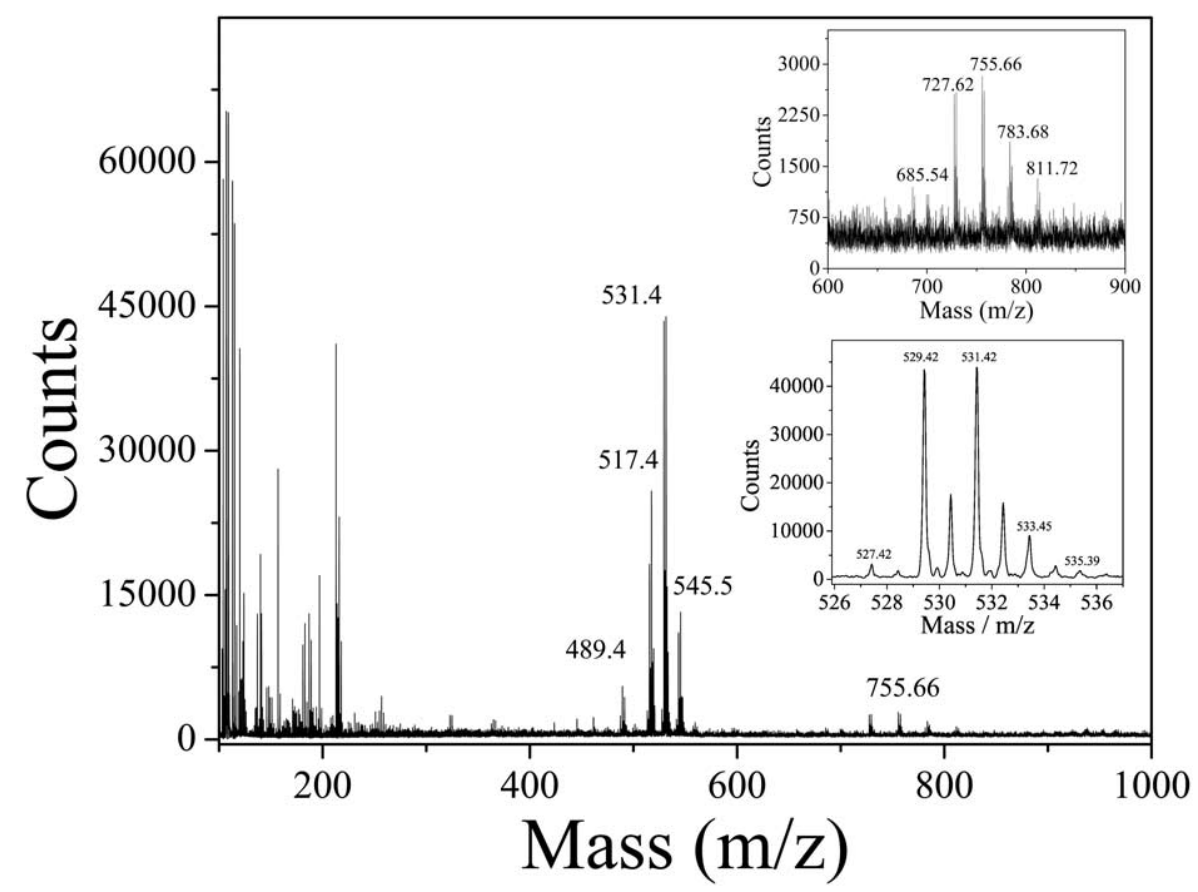

Figure 5. LDI-TOF mass spectrum obtained directly from the stem of Arabidopsis thaliana. The upper inset shows an enlarged view of the peaks for the ${ }^{107} \mathrm{Ag}$ adducts of esters. The lower inset shows an enlarged view of the peaks around $529.4 \mathrm{~m} / \mathrm{z}$. 
Table 1. Comparison of the relative amounts of various compounds in wax measured by LDI- TOF and by traditional methods

\begin{tabular}{|c|c|c|c|c|}
\hline \multirow[b]{2}{*}{$m / z$ range } & \multicolumn{2}{|c|}{ Wax compounds } & \multirow{2}{*}{$\begin{array}{l}\text { LDI-TOF } \\
\text { measurements } \\
\text { (\% of total) }\end{array}$} & \multirow[b]{2}{*}{$\begin{array}{l}\text { Literature dat } \\
\text { (\% of total) }\end{array}$} \\
\hline & Compound & $\begin{array}{c}m / z \text { of }{ }^{107} \mathrm{Ag} \\
\text { adduct }\end{array}$ & & \\
\hline \multirow[t]{3}{*}{$487-491$} & $\mathrm{C}_{27}$ alkane & 487.3 & $6.4 \pm 1.6$ & $2 \pm 0.3$ \\
\hline & $\mathrm{C}_{26}$ alcohol & 489.3 & & \\
\hline & $\mathrm{C}_{26}$ aldehyde & 487.3 & & \\
\hline \multirow[t]{3}{*}{$515-519$} & $\mathrm{C}_{29}$ alkane & 515.4 & $26.7 \pm 4.5$ & $48.8 \pm 1.8$ \\
\hline & $\mathrm{C}_{28}$ alcohol & 517.4 & & \\
\hline & $\mathrm{C}_{28}$ aldehyde & 515.3 & & \\
\hline \multirow[t]{3}{*}{$529-533$} & $\mathrm{C}_{29}$ ketone & 529.4 & $51.8 \pm 5.3$ & $43.4 \pm 2.2$ \\
\hline & $\mathrm{C}_{28}$ fatty acid & 531.3 & & \\
\hline & $\mathrm{C}_{29}$ alcohol & 531.4 & & \\
\hline \multirow[t]{3}{*}{$543-547$} & $\mathrm{C}_{31}$ alkane & 543.4 & $13.5 \pm 5.1$ & $5.0 \pm 0.7$ \\
\hline & $\mathrm{C}_{30}$ alcohol & 545.4 & & \\
\hline & $\mathrm{C}_{30}$ aldehyde & 543.4 & & \\
\hline \multirow[t]{2}{*}{$557-561$} & $\mathrm{C}_{31}$ ketone & 557.4 & $1.5 \pm 0.8$ & $0.8 \pm 1.3$ \\
\hline & $\mathrm{C}_{30}$ acid & 559.4 & & \\
\hline
\end{tabular}

proximately 200 ppm, which is in good agreement with specifications of this mass spectrometer when using external calibration. Ion peaks of wax compounds with masses larger than $400 \mathrm{~m} / \mathrm{z}$ are present. In addition, silver ions, clusters and various lowmass fragments are also detected. Results of the wax extract for the $m / z 400-800$ were nearly identical to those of the plant samples. This indicates that the analysis of cuticular waxes by LDI-TOF does not require prior extraction from plant material.

It should be noted that in order to produce good ion signal from LDI-TOF measurements, the plant tissue usually required $20-30 \%$ higher laser powers compared to that used for the extract. In some cases good signals from plant tissue could be obtained at a much lower laser power, the threshold being approximately 50\% (90 $\mu \mathrm{J}$ energy per laser pulse) of the maximum for this instrument. Lower laser powers usually gave less fragmentation in the $100-300 \mathrm{~m} / \mathrm{z}$ region. Fragmentation in the $400-800 \mathrm{~m} / \mathrm{z}$ region occurred when high laser powers $(>130 \mu \mathrm{J}$ per pulse) were used.

In Figure 5, the various peaks in the region of $480-560 \mathrm{~m} / \mathrm{z}$ correspond to the most abundant compounds of cuticular waxes of Arabidopsis thaliana [2, 3, $21,22]$. Figure 5 shows only four major peaks within this mass range. It is well known, however, that cuticular waxes are composed of a large number of lipid compounds [2, 3, 21, 22]. Many of these compounds have similar molecular masses. Thus, overlap of ion signals exists. This is best illustrated in the lower inset of Figure 5, which shows an enlarged view of the peaks around $529.4 \mathrm{~m} / \mathrm{z}$. The two large peaks are the silver-adduct ions and the smaller ones are related to various carbon and hydrogen isotopes. The pattern for these four peaks is similar to Figure 3 of the pure n-alkane compounds. However, there exist additional peaks in Figure 5 (with $\mathrm{m} / \mathrm{z}$ 533.4535.4). These are probably attributable to the overlap of ions from other compounds in wax. Indeed, according to the literature [3, 21, 22], $\mathrm{C}_{29}$ ketone (529.4 $\mathrm{m} / \mathrm{z}$ with the ${ }^{107} \mathrm{Ag}$ adduct) and $\mathrm{C}_{29}$ alcohol $(531.4 \mathrm{~m} / \mathrm{z}$ with the ${ }^{107} \mathrm{Ag}$ adduct) have similar abundances in the cuticular wax of Arabidopsis thaliana.

In order to assess the ability of LDI-TOF spectra to accurately represent the true composition of wax, our measurements were compared with literature data [3, 21, 22]. For the latter, traditional extraction procedures and GC-MS were used. We performed LDI-TOF analysis of ten individual tissue samples taken from various plants. The percentage of ion signal at each mass region was calculated based on the total intensities of the peaks from 489.4 to $557.4 \mathrm{~m} / \mathrm{z}$.

The LDI-TOF data in Table 1 represent good sampleto-sample reproducibility. The rather large standard deviations in the LDI-TOF data, when compared to the results from traditional methods, are probably related to variations in cuticular wax content of the individual tissue samples. Moreover, since LDI-TOF probes a small sample area, the signal is dependent on the cuticular wax content at the particular location of the laser beam. Such local variations in cuticular wax content may in fact be interesting but cannot be detected by using traditional extraction-based methods, where the averaged content is measured.

According to Table 1, the main difference in the results from LDI-TOF and traditional methods is in the 515-519 $\mathrm{m} / \mathrm{z}$ region. $\mathrm{C}_{29}$ alkane and $\mathrm{C}_{28}$ alcohol and aldehyde are the relevant compounds in this region according to the literature [3, 21, 22]. Moreover, in this region, $\mathrm{C}_{29}$ alkane accounts for approximately $94 \%$ of the total material in this mass range. Thus, the lowerthan-expected total signal in this region may be explained by the low ionization efficiency of the $C_{29}$ alkane, as discussed earlier (Figure 4). This produces an apparent increase in the relative amounts of other 


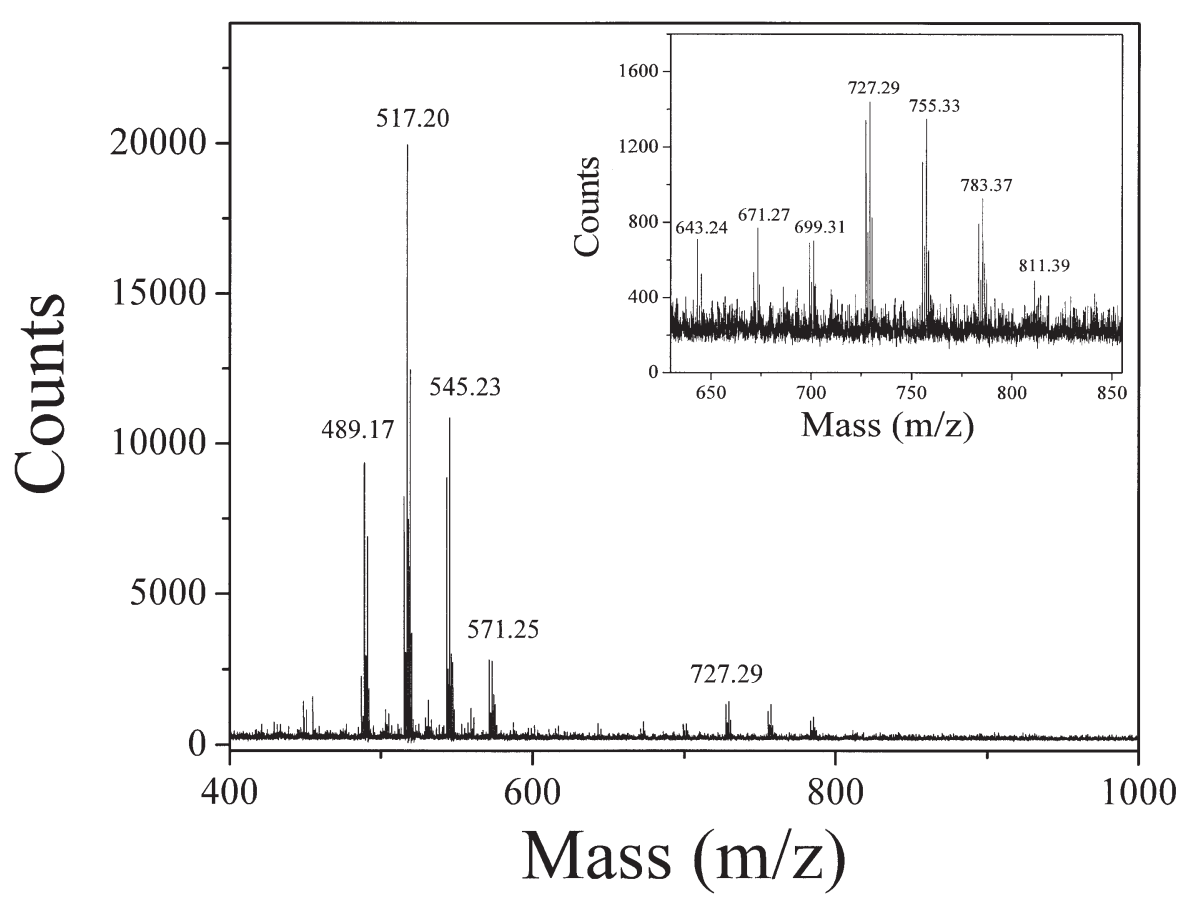

Figure 6. LDI-TOF mass spectrum obtained directly from the leaf of Arabidopsis thaliana. The inset shows an enlarged view of the peaks for the ${ }^{107} \mathrm{Ag}$ adducts of esters.

compounds in the mixture, in particular those with $\mathrm{m} / \mathrm{z}$ of 487-491 and 543-547.

Cuticular waxes also contain a wide variety of esters [2]. According to the literature [2], esters in cuticular wax range from $\mathrm{C}_{36}-\mathrm{C}_{64}$. The peaks at $729.6-811.7 \mathrm{~m} / \mathrm{z}$ in Figure 5 can therefore be attributed to the $\mathrm{C}_{42}-\mathrm{C}_{48}$ esters of cuticular waxes. The origin of the peak at 685.5 $\mathrm{m} / \mathrm{z}$ is unclear. The relative signal of the esters in Figure 5 (as percent of total of all peaks in Figure 5) was approximately $5-7 \%$. Literature data for Arabidopsis thaliana stems, however, indicates only $1-2 \%$ esters in cuticular wax [2]. This variation may be due to a combination of biological and/or developmental differences between the samples that were analyzed and the high ionization efficiency of esters in LDI-TOF.

Results for the analysis of an Arabidopsis thaliana leaf by LDI-TOF are given in Figure 6. Clearly, some differences do exist between the spectrum of Arabidopsis stem (Figure 5) and that of a leaf (Figure 6). In particular, ions around $517.2 \mathrm{~m} / \mathrm{z}$ are more prominent in Figure 6 and ions in the region of $531.4 \mathrm{~m} / \mathrm{z}$ are hardly detected. The former is consistent with the fact that the $\mathrm{C}_{29}$ alkane is the main compound in this mass range, accounting for approximately $40-45 \%$ of all materials in cuticular waxes of Arabidopsis thaliana [2, 3, 21, 22]. However, in leaves from Arabidopsis thaliana, the $\mathrm{C}_{29}$ alkane content decreases to less than $20 \%$ of the total materials [3]. In addition, the $\mathrm{C}_{28}$ alcohol $(517.4 \mathrm{~m} / \mathrm{z})$ content increases from $2 \%$ in the stem to approximately $10 \%$ for the leaf. Since the ionization efficiency of alcohols was found to be greater than that of alkanes (Figure 4), the overall effect is an increase of ion intensity at this particular mass range. The very low ion signal of the $531.4 \mathrm{~m} / \mathrm{z}$ peak can be explained by the difference in the $\mathrm{C}_{29}$ ketone in stem and leaf. The relative amount of this compound has been reported to decrease from $28 \%$ in stem to approximately $2 \%$ in leaf [3].

Results for the analysis of waxes from maize by LDI-TOF are given in Figure 7 and were compared to those of GC-MS (results not shown). The spectrum in Figure 7 indicates the existence of one major peak with a mass of $466.5 \mathrm{~m} / z(573.1 \mathrm{~m} / \mathrm{z}$ being the adduct of ${ }^{107} \mathrm{Ag}$ ). The peak corresponds to the $\mathrm{C}_{32}$ alcohol, which is a known major constituent of maize cuticular waxes. The mass spectrum of $\mathrm{C}_{32}$ alcohol (lower inset of Figure 7) appears very similar to that of the pure n-alkane standard mixture (Figure 3). However, it does not resemble the multi-peak pattern in the lower inset of Figure 5. Thus, LDI-TOF analysis of maize indicates the presence of only a single major compound in wax. This finding contradicts GC-MS analysis that indicates the existence of the $C_{32}$ aldehyde $(571.1 \mathrm{~m} / \mathrm{z})$ as another major compound in maize.

\section{Conclusions}

In this study, we examined the feasibility of laser desorption/ionization time-of-flight (LDI-TOF) mass spectrometry for the in situ analysis of cuticular wax compounds on the surface of intact plant material. We found that simple addition of colloidal silver onto the tissue surface gave good spot-to-spot reproducibility. Nearly identical mass spectra were acquired from plant tissue and from its extract. Thus, we confirm that analysis of waxes by LDI-TOF does not require prior 


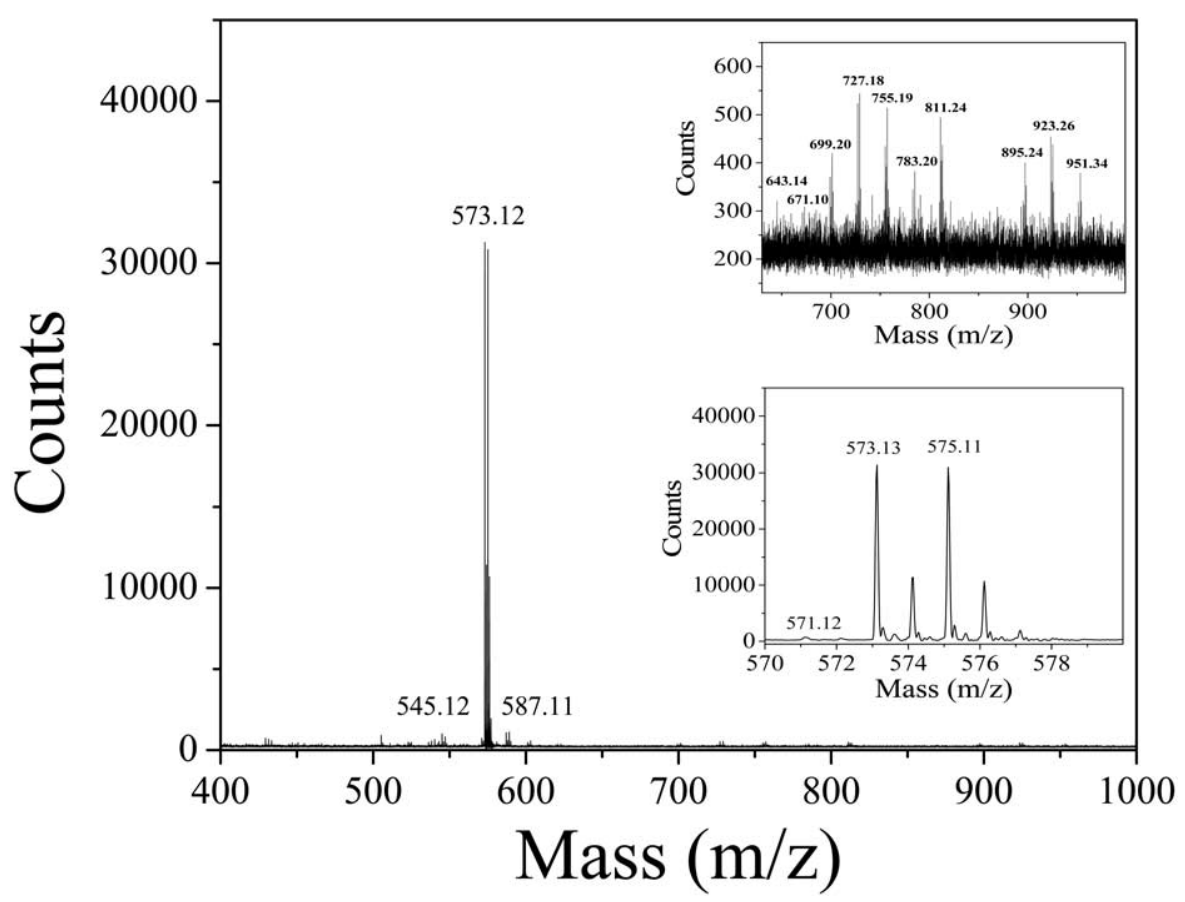

Figure 7. LDI-TOF mass spectrum obtained directly from maize. An enlarged view for peaks of esters is given in the upper inset. The lower inset shows the enlarged view for the peak at $573.12 \mathrm{~m} / \mathrm{z}$. Masses in the insets correspond to the ${ }^{107} \mathrm{Ag}$ adduct.

extraction from plant material. Although the results indicate that this method is biased towards compounds of high ionization efficiency, it does provide a good estimate of the relative abundance of compounds in waxes. Some overlap of the ion peaks from the various compounds in cuticular waxes exists. These can probably be resolved by tandem mass spectrometry or by improving the mass resolution of measurements. At this stage, LDI-TOF provides rapid and highly efficient analysis of waxes directly on plant material and can therefore be used for high-throughput screening purposes. The method is simple, fast, and does not require special sample pretreatment prior to analysis. Because of the homogeneous distribution of the silver colloids, LDI-TOF has the potential to be used for measuring the composition at different locations on the plant, i.e., for imaging applications.

\section{Acknowledgments}

ESY thanks the Robert Allen Wright Endowment for Excellence for support. The Ames Laboratory is operated for the U.S. Department of Energy by Iowa State University under contract no. W-7405-Eng-82. This work was supported by the Director of Science, Office of Basic Energy Sciences, Division of Chemical Sciences.

\section{References}

1. Sumner, L. W.; Mendes, P.; Dixon, R. A. Plant metabolomics: Large-scale phytochemistry in the functional genomics era. Phytochemistry 2003, 62, 817-836.
2. Kunst, L.; Samuels, A. L. Biosynthesis and secretion of plant cuticular wax. Prog. Lipid Res. 2003, 42, 51-80.

3. Rashotte, A. M.; Jenks, M. A.; Feldmann, K. A. Cuticular waxes on eceriferum mutants of Arabidopsis thatiana. Phytochemistry 2001, 57, 115-123.

4. Kuhn, G.; Weidner, S.; Just, U.; Hohner, G. Characterization of technical waxes. Comparison of chromatographic techniques and matrix-assisted laser-desorption/ionization mass spectrometry. J. Chromatogr. A 1996, 732, 111-117.

5. Chen, R.; Li, L. Reactions of atomic transition-metal ions with long-chain alkanes. J. Am. Soc. Mass Spectrom. 2001, 12, 367375.

6. Pruns, J. K.; Vietzke, J. P.; Stranssner, M.; Rapp, C.; Hintze, U.; Konig, W. A. Characterization of low molecular weight hydrocarbon oligomers by laser desorption/ionization timeof-flight mass spectrometry using a solvent-free sample preparation method. Rapid Commun. Mass Spectrom. 2002, 16, 208-211.

7. Dutta, T. K.; Harayama, S. Time-of-flight mass spectrometric analysis of high-molecular-weight alkanes in crude oil by silver nitrate chemical ionization after laser desorption. Anal. Chem. 2001, 73, 864-869.

8. Karas, M.; Hillenkamp, F. Laser desorption ionization of proteins with molecular masses exceeding 10,000 Daltons. Anal. Chem. 1988, 60, 2299-2301.

9. Karas, M.; Ingendoh, A.; Bahr, U.; Hillenkamp, F. Ultravioletlaser desorption/ionization mass spectrometry of femtomolar amounts of large proteins. Biomed. Environ. Mass Spectrom. 1989, 18, 841-843.

10. Yoshida, T.; Tanaka, K.; Ido, Y.; Akita, S.; Yoshida, Y. High molecular ion detection using laser ionization TOF mass spectrometer. 35-kai Shitsuryo Bunseki Rengo Toronkai, Yoshishu; Japan. 1987, pp 30-31.

11. Tanaka, K.; Ido, Y.; Akita, S.; Yoshida, Y.; Yoshida, T. Detection of high mass molecules by laser desorption time-of-flight 
mass spectrometry; Proceedings of the Second Japan-China Joint Symposium on Mass Spectrometry; 1987pp 185-188.

12. Yalcin, T.; Wallace, E. W.; Guttman, C. M.; Liang, L. Metal powder substrate-assisted laser desorption/ionization mass spectrometry for polyethylene analysis. Anal. Chem. 2002, 74, $4750-4756$.

13. Trimpin, S.; Rouhanipour, A.; Az, R.; Rader, H. J.; Mullen, K. New aspects in matrix-assisted laser desorption/ionization time-of-flight mass spectrometry: A universal solvent-free sample preparation. Rapid Commun. Mass Spectrom. 2001, 15, $1364-1373$.

14. Trimpin, S.; Grimsdale, A. C.; Rader, H. J.; Mullen, K. Characterization of an insoluble poly(9,9-diphenyl-2,7-fluorene) by solvent-free sample preparation for MALDI-TOF mass spectrometry. Anal. Chem. 2002, 74, 3777-3782.

15. Li, L.; Garden, R. W.; Sweedler, J. V. Single-cell MALDI: A new tool for direct peptide profiling. Trends Biotechnol. 2000, 18, 151-160.

16. Rubakhin, S. S.; Greenough, W. T.; Sweedler, J. V. Spatial profiling with MALDI MS: Distribution of neuropeptides within single neurons. Anal. Chem. 2003, 75, 5374-5380.
17. Stoeckli, M.; Chaurand, P.; Hallhan, D. E.; Caprioli, R. M. Imaging mass spectrometry: A new technology for the analysis of protein expression in mammalian tissues. Nat. Med. 2001, 7, 493-496.

18. Stahl, B.; Linos, A.; Karas, M.; Hillenkamp, F.; Steup, M. Analysis of fructans from higher plants by matrix-assisted laser desorption/ionization mass spectrometry. Anal. Biochem. 1997, 246, 195-204.

19. Miller, J. C.; Haglund, R. F. Laser Ablation and Desorption; Academic Press: New York, 1998, pp 1-76.

20. Riederer, M.; Schreiber, L. Protecting against water loss: Analysis of the barrier properties of plant cuticles. J. Exp. Botan. 2001, 52, 2023-2032.

21. Millar, A. A.; Clemens, S.; Zachgo, S.; Giblin, E. M.; Taylor, D. C.; Kunst, L. CUT1, an Arabidopsis gene required for cuticular wax biosynthesis and pollen fertility, encodes a very-long-chain fatty acid condensing enzyme. Plant Cell 1999, 11, 825-838.

22. Jenks, M. A.; Tuttle, H. A.; Eigenbrode, S. D.; Feldmann, K. A. Leaf epicuticular waxes of the Eceriferum mutants in Arabidopsis. Plant Physiol. 1995, 108, 369-377. 